\title{
CORRECTIONS
}

\section{Bring in universal mental health checks in schools}

The author's position in this Personal View (BMJ 2013;347:f5478, doi:10.1136/bmj.f5478) is not fully correct. Simon N Williams is currently a visiting scholar at the Feinberg School of Medicine at Northwestern University, Chicago. However, Williams was formerly a research associate at the Institute of Public Health at the University of Cambridge; he was not an academic clinical fellow at the university, as was published.

Cite this as: BMJ 2013;347:f6117

๑ BMJ Publishing Group Ltd 2013 